\title{
Cooperation, trust, and social capital in Southeast Asian urban slums
}

\author{
Jeffrey P. Carpenter ${ }^{\mathrm{a}, *}$, Amrita G. Daniere ${ }^{\mathrm{b}}$, Lois M. Takahashi ${ }^{\mathrm{c}}$ \\ ${ }^{\text {a }}$ Department of Economics, Middlebury College, Middlebury, VT, USA \\ ${ }^{\mathrm{b}}$ Geography and Planning, University of Toronto, Toronto, Ont., Canada \\ ${ }^{\mathrm{c}}$ Department of Urban Planning, University of California, Los Angeles, CA, USA
}

Received 19 May 2003; received in revised form 28 October 2003; accepted 13 November 2003

Available online 31 July 2004

\begin{abstract}
We conduct experiments in urban slums to measure trust and cooperation and to see how behavior varies with demographic factors and associational measures of social capital. Overall, we find high contribution rates among Thai and Vietnamese participants in a voluntary contribution game, and we see that many participants are willing to signal their disapproval of free riding despite it being costly to do so. At the individual level, we find that behavior varies with many demographic factors and with many associational factors. However, these correlations often differ significantly between our two locations, indicating the role of culture, defined broadly.

(c) 2004 Elsevier B.V. All rights reserved.
\end{abstract}

JEL classification: $\mathrm{C} 91 ; \mathrm{C} 92 ; \mathrm{H} 41 ; \mathrm{I} 32$

Keywords: Cooperation; Trust; Social disapproval; Thailand; Vietnam

\section{Introduction}

A current trend in the economic development literature is to identify and understand how social aspects of individual and community behavior contribute to (or detract from)

\footnotetext{
* Corresponding author. Tel.: +1 802443 3241; fax: +1 8024432084.

E-mail addresses: jpc@middlebury.edu (J.P. Carpenter), amrita.daniere@utoronto.ca (A.G. Daniere), takahash@sppsr.ucla.edu (L.M. Takahashi).
}

0167-2681/\$ - see front matter (C) 2004 Elsevier B.V. All rights reserved. doi:10.1016/j.jebo.2003.11.007 
economic performance. ${ }^{1}$ Much of this literature has been grouped under a poorly defined term: social capital. The term is poorly defined because, to one set of researchers, social capital is defined as the propensities of individuals to trust, cooperate, and punish other individuals who act to establish and maintain prosocial norms of behavior (e.g., Fukuyama, 1995; Bowles and Gintis, 2002; Glaeser et al., 2002). At the same time, however, to other researchers social capital means the community level networks among individuals that lead to efficient outcomes when contracts are hard to enforce (e.g., Putnam, 2000). Despite an outpouring of research using both of these definitions of social capital, there remains significant conceptual confusion regarding the aspects of communities and individuals that demonstrate social capital. To be more specific, we think of the first definition as behavioral social capital and the second as associational social capital. One of our purposes is to search for links between these two concepts.

As if conceptual problems were not enough to overcome, social capital research is also confounded by measurement and estimation issues (Durlauf, 2002). Specifically, different definitions of social capital lead to different strategies for measuring its effects. At the individual level, researchers look for behavioral measures of trust, trustworthiness, and cooperation, all in the (implied) context of social dilemmas where individual incentives are at odds with collective efficiency. These measures typically come from surveyed self-reports of behavior and attitudes. A representative question from the general social survey (GSS) is “Generally speaking, would you say that most people can be trusted, or that you can't be too careful in dealing with people?" Research that focuses on the associational definition of social capital, while also typically based on self-reports from surveys, asks less hypothetical and more factual questions, such as "How many volunteer organizations do you belong to?"

It is not news that surveys are an imperfect way to gather information about individual behavior, but their practical advantages (i.e., they are comparatively cheap to conduct and often provide a large sample) continue to make them popular research tools. The benefits of surveys, however, may be outweighed in the case of behavioral social capital. While there surely exists measurement error in the more factual network questions, if for no other reason than because respondents are careless, hypothetical questions about behavior add other biases that may be more worrisome because they tend to be systematic. As just one example, who wants to think of him or herself as untrustworthy? ${ }^{2}$

Our second purpose is to report on an attempt to measure behavioral social capital in the field using experiments instead of surveys. We hypothesize that experiments provide more accurate measures of behavioral social capital because paying participants based on their choices provides an incentive to take the exercise seriously (especially when the stakes are high) and because participants must risk money to trust or cooperate. That is, providing incentives for our participants should reduce much of the "noise" associated with hypothetical survey measures of prosocial behavior.

Our study is unique for four reasons. First, while much of the work on social capital is focused on residents of the industrialized west, our participants are from Southeast Asia.

\footnotetext{
${ }^{1}$ Examples include Desdoigts (1999), Knack and Keefer (1997), and Woolcock (1995). The near exponential growth of such research is documented in Isham et al. (2002).

2 See Carpenter (2002a) and Bertrand and Mullainathan (2001) for an expanded discussion of the difficulties of using surveys to elicit behavioral data.
} 
Second, instead of using students, our participants live and work in urban slums. Specifically, our research brought us to five communities in Bangkok and five communities in Ho Chi Minh City. We find these populations particularly interesting because people in the developing world often face social dilemmas on a daily basis and therefore may behave differently in situations that require trust or cooperation than students in the industrialized west. With this fact in mind, our results should be more directly relevant for studies of poverty and development. ${ }^{3}$ Third, instead of using the popular Berg et al. (1995) investment game to measure trust and cooperation (as in Ashraf et al., 2003; Croson and Buchan, 1999; Carter and Castillo, 2002), we employ a voluntary contribution experiment (like Gächter et al., 2003) in which cooperative acts measure the degree to which participants trust that other participants will also cooperate. Fourth, because we also gathered associational data in a post-experiment interview, we examine the links between associations and behavior.

\section{Background and community descriptions}

Our primary interest in slum dwellers, the cities of Bangkok and Ho Chi Minh, and this region in general, is that the area is experiencing rapid growth and urbanization while a significant portion of the population is increasingly marginalized. The resulting increase in inequality, specifically, is the source of serious concern for policy makers in the region (Fritzen, 2002; Kakwani and Krongkaew, 2000). Many marginalized households end up in urban slums where economic opportunities are scarce and health and environmental problems are abundant. We are interested in the determinants of trust and cooperation under these circumstances for policy reasons because, increasingly, policy in developing countries presumes some predisposition to cooperate on the part of the poor.

Southeast Asia is a region composed of several economic tigers surrounded by a few countries where little has changed or improved in the past half century (e.g., Cambodia, Laos and Myanmar/Burma). ${ }^{4}$ Thailand and Vietnam, however, represent relative success stories in this rapidly industrializing part of the developing world although their paths to success could not, at least until recently, have been more different. Aside from the basic similarities of religion, size of population, geographic endowments, and so on., the political economy of the two nations differ in many, if not all, respects.

Thailand, and specifically Bangkok, has adopted a generally laissezfaire approach to economic development in the last 30 years. As such, the government of Thailand has permitted and, some argue, even encouraged the extreme concentration of industrial, manufacturing, commercial and service sectors within the Bangkok Metropolitan Region. While several steps have been taken since the early 1990s to support the deconcentration of economic activities to smaller cities within 100 or so miles of Bangkok, these efforts have had relatively little effect on Bangkok's rate of growth.

\footnotetext{
${ }^{3}$ For evidence supporting this view see Henrich et al. (2001) who show that the variance in behavior in a simple bargaining experiment conducted in the field in fifteen small-scale societies can be explained by the extent to which cooperation is needed in local production.

4 There is increasing debate regarding the actual level of success of so-called tiger economies of Thailand, Indonesia and Malaysia given the relatively limited growth these countries are still experiencing 6 years after the beginning of the Asian crisis (Singh and Freeman, 2001).
} 
Bangkok has expanded exponentially since the end of the Vietnam War era and now encompasses about 12 million people (out of a total population of 64 million). The land mass occupied by these residents and their places of employment extends far beyond the traditional city core and into vast suburban and even rural landscapes. The city itself is extremely dense, congested and dirty, although Bangkok's Metropolitan Authority (the municipal government) has attempted, with the support of the central government, to ease traffic, improve sanitation, and deliver cleaner water. The lack of an effective means of enforcement of environmental and economic regulations, however, as well as a reluctance on the part of the Thai government to invest in physical and social infrastructure, has contributed to the relatively unfortunate situation of hundreds of thousands of slum dwellers.

Recent media accounts indicate a growing public dissatisfaction with environmental conditions, expressed through public protests focused on air and water pollution. Local mobilization (such as urban squatter associations, loosely organized through NGOs and/or community groups) can, in specific circumstances particularly with the assistance of wellplaced contacts in the Bangkok Metropolitan Authority or one of the national level ministries, achieve substantial improvements in local quality of life. Squatter settlements have obtained piped water connections, structural upgrades or day care centers as well as improved land tenure arrangements through local organization and lobbying of key bureaucrats. Thus, social capital within communities could potentially be of great value to slum dwellers in terms of the potential of networks and trust to encourage economic and community development (Daniere et al., 2002).

Vietnam, however, is very different. The political economy of Vietnam has transformed since 1986 and the introduction of doi moi (meaning renovation but actually describing the liberalization process). The center of economic growth in Vietnam is Ho Chi Minh City. Although slightly more than one-third the size of Bangkok, Ho Chi Minh City has experienced extremely rapid expansion, leading to its characterization as the world's next "Bangkok" (Drakakis-Smith and Dixon, 1997). There has been widespread expansion in the urban area, and many additional households have moved to the city to be closer to opportunities even without the paperwork required to dwell legally inside the metropolis.

While the Vietnamese communist party tolerates neither any public advocacy of ideological or political pluralism nor any citizen's criticism of its foreign policies, the relationship between the state and society in Vietnam is considerably more dynamic and changing more rapidly than is generally perceived. In particular, non-registered locally based voluntary associations have sprung up all over the country in the last 15 years. They include samevillage or same-province associations in the large cities, alumni, same-military-service, rotating credit associations, and so on. While none of these new associations are political in their orientation, the ties formed through them have been mobilized for collective action and for a concerted voice for better local governance (Luong, 2003). As such, community groups and ward-level People's Committees are beginning to have a say in how local government spending is allocated within their boundaries. Hence, social capital and the ability to organize and work together to initiate local change is very much part of the urban landscape in Vietnam.

While there are many differences in the institutions, economics, and cultures between the two cities, the challenges faced are somewhat similar because of two factors: Vietnam 
will likely continue to depend on doi moi to help jump start its economy, and both Thailand and Vietnam are increasingly exposed to foreign investment and the consumer culture of the West apparent in most globalizing cities (Kim et al., 1997). Consequently, both national and urban governments will look for new and cost-effective strategies to sustain economic growth. Community participation and the role played by social capital remain key factors believed by international donor agencies, NGOs, and government agencies to be vital to the potential success of urban development and environmental policies.

To measure the level of social capital and trust in poor urban communities of Bangkok and Ho Chi Minh City, we conducted experimental games and a post experiment survey in five low-income communities in each city. ${ }^{5}$

In Bangkok, the communities were selected through the use of a sampling frame developed from a study of Bangkok slums (Setchell, 1992) and the local expertise of the project team and their contacts. The communities were not selected randomly but are generally representative of the broad range of slums and squatter areas in terms of size, history, location and environmental conditions that one might see in the Bangkok Metropolitan Region. The selection criteria included low average per capita or household income and disparate locations to ensure that there would be some variation in terms of access to services (see Daniere et al., 2002). We contacted the neighborhood organization or community leaders in each of the neighborhoods to request permission to work with the community. Each of the five communities we approached agreed to work with us. In the case of experimental games, potential participants were informed through leaflets and community announcements. Participants were asked to meet at a central location, generally the local community center or day care, if they wanted to volunteer for the games. The players were randomly selected from among the volunteers gathered at the site.

To select communities in Ho Chi Minh City, we needed to rely to a greater degree on local knowledge and connections. The slum communities could not be selected from a sampling frame but were instead proposed by the People's Committees responsible for specific city districts. We approached five different districts and asked them to nominate one or two of their most impoverished wards or communities for inclusion in the project. Since the research project has the support of the Vietnamese government and one of our project team members is a government employee at the Institute for Social Sciences, we were able to conduct our research relatively free of control or interference. It is possible, of course, that we were directed to showpiece communities although given our extensive travels throughout the city, this seems unlikely to us and to our Vietnamese colleagues.

The experiments in Ho Chi Minh City were more complex to organize than in Bangkok. We chose to conduct the games in meeting rooms at the Institute for Social Sciences to minimize neighborhood disruption and monitoring by party (People's Committee) officials. Participants from the different communities who volunteered for the experiment were transported by van or taxi to the center, played the game, and were then provided transportation back to their communities. As in Bangkok, potential participants were informed that the game would result in immediate earnings; there was no lack of volunteers.

\footnotetext{
5 The names, locations and brief descriptions of the different communities can be found together with the
} experimental instructions and our survey on line at http://community.middlebury.edu/ jcarpent/papers.html. 


\section{Our experimental protocol}

Our field experiments were conducted during the summer of 2002 and consisted of a hand-run version of the voluntary contribution mechanism in which players were organized into groups and individually decided how much to contribute to a public good.

For us, trust is the willingness to abandon strategic deliberation (in particular subgame perfection), making one vulnerable to others, in the belief that one will be better off as a result. With this definition in mind, a number of experimental games may be used to measure trust. As mentioned above, a popular experiment is the Berg et al. investment game in which a first mover sends money through the experimenter to a second mover. Any money that is sent is multiplied by a factor greater than one so that sending is socially efficient. The second mover can then send any fraction of the amount she receives back to the first mover. In this context, the amount sent by the first mover is thought to measure trust. There are also a number of discrete choice games with incentives that are similar to the investment game (e.g., McCabe et al., 1996; Güth et al., 1997).

In psychology, there has been a tradition of associating trust with being cooperative in the prisoner's dilemma (see Deutsch, 1973) and this area of research has, more recently, been explored by economists and political scientists (e.g., Ahn et al., 2003). Indeed, experimental studies have established a link between trust and cooperative acts in two-person prisoner's dilemma games (Messick et al., 1983; Parks and Hulbert, 1995). Based on this research, we chose to study trust by implementing the voluntary contribution game as the four-person generalization of the prisoner's dilemma.

We gathered data from 240 participants (120 from each city). Our 240 participants were split into 60 four-person groups, and the composition of the groups remained the same for the entire experiment. This is known as the partners protocol. There were six groups from each of the 10 communities. Our procedures are interesting from an experimental point of view because, unlike most other experiments, we controlled for the gender composition of our groups. In each community, we formed two all male groups, two all female groups, and two half male half female groups.

The experiment typically lasted less than 2 hours including instructions, payment, and an exit survey. We were concerned about the size of the potential earnings, so we calibrated the payoffs at the social optimum to be between one-half and slightly more than one week's wage (based on local industrial wages that were approximately US\$ 44 in Thailand and US\$ 12 in Vietnam). In practice, our Thai players earned US\$ 21.62 and our Vietnamese players earned US\$12.42, on average.

The experiment consisted of two treatments and a total of 10 rounds. In the first five rounds, participants played a standard voluntary contribution game, and in the second five rounds, the game was modified to allow players to socially sanction (i.e., show their disapproval of) free riders. This game is interesting because it allows us to assess cooperation and trust by the levels of contribution, but the game also allows us to assess players' willingness to express their dissatisfaction with the contributions of others.

The details of our experimental procedures are as follows. Before we began the experiment, the instructions and game materials were translated into the local language, then the back translations were discussed to iron out any contextual problems. During the experiment these instructions were presented orally by the same experimenter in each location to 
control for experimenter effects. ${ }^{6}$ The instructions contained a few examples that participants could ask questions about to assure that they understood the game. During the first five rounds each player was endowed with 10 monetary units: ten 1000 Dong bills in Vietnam or ten 5 Bhat coins in Thailand. ${ }^{7}$ One at a time, each member of a group walked behind a blind set up to make decisions as anonymous as possible. Once behind the blind, each group member contributed as much of her 10-unit endowment as she wanted to a "group project." Each player then placed the rest of her endowment in an opaque, color-coded envelope and returned to her seat.

Once all the group members made their contributions, one of the experimenters wrote each of the four contributions on a slip of paper in random order (to keep them anonymous) and calculated the sum. One at a time, the players then returned behind the blind to see how much had been contributed individually and collectively to the group project. At this point each person in the group received her payoff from the group project, which was an equal share of the doubled sum of the group contributions. Each person put her share of the group project in her envelope and then returned to her seat. This process was repeated five times.

Our procedures create a social dilemma for the subjects because each has a material incentive to free ride on the contributions of others. The per period payoff to player $i$ who contributes $x_{i}$ is

$$
\pi_{i}=\left(10-x_{i}\right)+\frac{2 \sum}{4} x_{i}
$$

which implies that every monetary unit contributed returns only half a unit to the contributor; therefore, contributing zero is the dominant strategy. However, this a dilemma because if all participants contribute fully, everyone in the group receives 20 monetary units instead of the 10 they receive if everyone uses the dominant strategy. Hence, $x_{i}$ is a behavioral measure of a participant's propensity to trust and cooperate in the face of the material incentive to not cooperate.

Rounds 6 through 10 were conducted exactly like rounds one through five except for one proceedural change. ${ }^{8}$ Now, when players returned behind the blind to see what everyone had contributed and to pick up their share of the group project, they were given the opportunity to sanction the rest of the group if they did not like the group's contribution profile. Specifically, each player was asked whether or not she wanted to have a picture displayed that meant she was unhappy with what the others had contributed. The picture was meant to be easy to interpret. We chose an unhappy face.

It was costly to have the picture displayed so that, like contributing, showing disapproval was dominated by remaining silent. It cost 200 Dong in Vietnam and 1 Bhat in Thailand to display a picture. Any purchased pictures were displayed at the beginning of the next round so that the sources of the pictures were anonymous. This procedure means that players saw between zero and four pictures when they made their next contribution decisions.

\footnotetext{
6 Anchana Na-Ranong in Bangkok and Van Thi Ngoc Lan in Ho Chi Minh City.

7 At the time of the experiment the Dollar-Dong exchange rate was approximately US $\$ 1=15,000 \mathrm{VND}$ and the Dollar-Bhat exchange rate was approximately US\$ $1=40$ Bhat.

8 The players did not know that the rules would change until after round 5 was completed. We did this to prevent any confounds associated with players anticipating the rule change.
} 
Table 1

Demographics, social capital indices, and experimental behavior

\begin{tabular}{|c|c|c|c|c|c|c|}
\hline & \multicolumn{3}{|c|}{ Bangkok } & \multicolumn{3}{|c|}{ Ho Chi Minh City } \\
\hline & Obs. & Mean & S.D. & Obs. & Mean & S.D. \\
\hline \multicolumn{7}{|l|}{ Demographics } \\
\hline Participant sex $(1=$ male $)$ & 120 & 0.50 & 0.50 & 120 & 0.50 & 0.50 \\
\hline Participant years of schooling & 120 & 6.92 & 1.14 & 120 & 9.67 & 3.42 \\
\hline Participant age & 120 & 40.98 & 13.71 & 120 & 35.10 & 13.69 \\
\hline Household size & 119 & 4.97 & 2.43 & 120 & 5.43 & 2.44 \\
\hline Years of slum residence & 119 & 18.24 & 13.20 & 118 & 21.82 & 13.73 \\
\hline Cooperation scale (6 (high) to -6 (low)) & 120 & 2.80 & 0.56 & 120 & 2.34 & 0.47 \\
\hline \multicolumn{7}{|l|}{ Associational social capital } \\
\hline Own home $(1=$ yes $)$ & 120 & 0.76 & 0.43 & 120 & 0.92 & 0.28 \\
\hline Community homogeneity ( 1 = high) & 118 & 0.21 & 0.41 & 102 & 0.05 & 0.22 \\
\hline Participate in community project $(1=$ yes $)$ & 116 & 0.92 & 0.27 & 114 & 0.93 & 0.26 \\
\hline Chat (likert scale 1 (low) to 4 (high)) & 119 & 3.36 & 0.72 & 120 & 3.81 & 0.51 \\
\hline Describe (likert scale 1 (low) to 3 (high)) & 119 & 3.44 & 0.50 & 120 & 3.46 & 0.53 \\
\hline Leader of community $(1=$ yes $)$ & 120 & 0.17 & 0.37 & 120 & 0.07 & 0.25 \\
\hline \multicolumn{7}{|l|}{ Experimental behavior } \\
\hline Average cooperation in the experiment & 120 & 6.72 & 2.41 & 120 & 7.41 & 2.10 \\
\hline Fraction of individuals who disapprove & 120 & 0.34 & 0.47 & 120 & 0.25 & 0.43 \\
\hline
\end{tabular}

Because it was costly to sanction the other players in one's group, individuals could always do better by free riding on the sanctioning done by others. By backward induction, knowing that sanctioning is dominated, free riders should not fear sanctions, so the only subgame perfect equilibrium of this finitely repeated game is to continue to free ride. ${ }^{9}$ Despite the subgame perfect prediction, if players do sanction, it is a behavioral measure of their willingness to incur a cost to express their disapproval of others.

\section{Data overview}

Table 1 summarizes three aspects of our participants: their demographics, their associational social capital, and their behavior in our experiment. By design, our participants were half men and half women. On average, our Thai participants had finished grade school, and our Vietnamese participants had completed junior high school. This difference in educational attainment is significant $(t=8.36, p<0.01)$. Our participants are much older than the standard student population, and our Thai participants are significantly older than our Vietnamese participants $(t=3.32, p<0.01)$. In both locations our participants come from households with around five members. Our Thai participants had spent an average of 18.24 years in their slum community, and our Vietnamese participants had lived in their communities for 21.82 years, on average. These last statistics imply that our participants had spent approximately half their lives in the slums.

\footnotetext{
${ }^{9}$ Alternatively, notice that since sanctions impose no material harm on free riders, they should be ignored by payoff maximizing players.
} 
The last demographic statistic we will consider was formulated from three questions taken from two standard psychological scales used to measure cooperative predispositions, the AB5C: II+/I- and the NEO: A4. ${ }^{10}$ We included the cooperation scale to account for possible selection problems driven by cooperative personalities that might be distributed non-randomly. As it turns out, the Thais seem to have been more cooperatively predisposed $(t=4.50, p<0.01)$. Because we needed to keep the post-experiment survey brief, the scale consisted of adding the following three items that seemed most relevant for our current purposes ('+' means the item was scored positively for agreeing and '-' means the opposite $)^{11}$

1. It is better to cooperate than compete. $(+1)$

2. People should listen to their conscience when making decisions. $(+1)$

3. It is amusing to play tricks on other people. $(-1)$

In our post-experiment interview, we gathered data on our participants' associational social capital. The existing evidence suggests that home ownership provides people an anchor in the community they would not otherwise have (Sampson et al., 1997; Glaeser and Sacerdote, 1999). Own home is a dummy variable that takes the value of one for people who own their homes. Reported home ownership is significantly higher in Vietnam $(t=$ $3.42, p<0.01)$. We expected people who own their own homes would be more vested in the community and therefore more cooperative and more likely to show their disapproval of free riding. We expected that people from more homogeneous communities will be more cooperative with and more likely to punish other members of their "ingroup" (Messick and Mackie, 1989; Cardenas and Carpenter, 2002). Community homogeneity measures the respondent's perception of the regional composition of her community. Specifically, this question asked, "when new people come to your community, do they mostly come from the same village or region or do they come from many different places?" Regional homogeneity is perceived by participants to be particularly low in Vietnam and the inter-city difference is significant $(t=3.53, p<0.01)$.

Many of our participants organize projects each year to clean up or improve the conditions in their communities. Participation in these projects is voluntary; therefore people have the incentive to free ride. Participate in community project equals one if the respondent or someone in the respondent's family participated in the project. According to respondents in both countries participation in these projects is very close to 100 percent, which is inconsistent with information from casual conversations with community leaders. This inconsistency illustrates the problem with surveyed self-reports.

Chat is a likert scale response to the question: "How often do you chat or spend time together with other people in your community?" Describe, also measured on a likert scale, is the response to the question: "How do you describe your neighbors who are not relatives?" The responses to this question varied between (1) like strangers to (3) like family. Finally,

\footnotetext{
${ }^{10}$ Each of these two psychological scales have high Cornbach alpha values ( 0.73 for both) that measure the extent to which the scales capture a single unidimensional latent construct, cooperativeness in this case. The scales are available on line at http://ipip.ori.org.

11 While the reported Cornbach interitem correlation is high for each of the original scales, it is considerable lower in our implementation $(\alpha=0.34)$.
} 
we were also able to identify people who were politically active in the communities. Leader of community is a dummy which takes the value of one for anyone whom our collaborators identified as a community leader (e.g., ward leaders in Vietnam). We see that we recruited more leaders in Thailand $(t=2.45, p<0.02)$, the Vietnamese were more connected to their neighbors, on average $(t=5.58, p<0.01)$, and both groups described their neighbors as being mostly like family.

In terms of summary statistics, both groups of participants contributed at very high levels in our experiment. Our Thai participants' average contribution, pooling all 10 rounds, was 6.72 of their 10 coin endowment, and the Vietnamese contributed even more, 7.41 of their 10 bill endowment. The difference in aggregate behavior is significant $(t=2.36, p<0.02)$, indicating that the Vietnamese participants were generally more trusting and cooperative. Additionally, both groups signaled their disapproval of free riding at high levels given that doing so is costly and inflicts no material harm on the free riders. Approximately one-third of the Thai showed disapproval and a quarter of the Vietnamese disapproved at least once.

We were astonished by the high contribution rates and wondered if our field protocol, which framed the encounter as contributing to a community project, could be responsible for such high contribution rates. To test this hypothesis, we conducted nine control sessions using the same protocol with Middlebury College students. ${ }^{12}$ The Middlebury contribution data in the first five perods show the anticipated decline in contributions and the standard end-game effect, indicating that the protocol can not explain the high contribution rates among our slum dwellers. There is, however, a marked effect of the social disapproval treatment, at least initially. Contributions among Middlebury students increase substantially in anticipation of social disapproval and decline again at a slower pace. We also see the endgame drop in contributions at the end of the second five periods. The similarities between our Middlebury data and other student experiments suggests that our protocol is not responsible for the high contribution rates we see in our field communities.

\section{The link between behavioral and associational social capital}

Our experiment generated a panel of data from 240 individuals over 10 periods. To account for individual heterogeneity over time, we use the random effects model. Because contributions are bound from below by zero and from above by 10 , we use the tobit estimator for our trust/cooperation analysis. Lastly, because our social sanction variable is binary, we use the logit estimator for our analysis of why individuals show disapproval. We build our empirical models in two steps. In our initial regressions we included only individual demographics, and in the second set we added our measures of associational social capital to test for any links between behavior and the network connectedness of our participants.

Table 2 presents the results of our analysis of contributions in the two cities. Almost all the demographics play a significant role in determining contributions. In Bangkok, contrary to most people's prior expectations, men contribute significantly more than women, schooling appears to teach people to free ride because an additional year of education reduces one's

\footnotetext{
12 Again, we tried to balance the gender composition of our groups. We were only slightly less successful. Our sample of nine groups is composed of three all men groups, two all women groups, and four mixed groups.
} 
Table 2

Dependent variable is an individual's contribution in a given round (results are random effects Tobit)

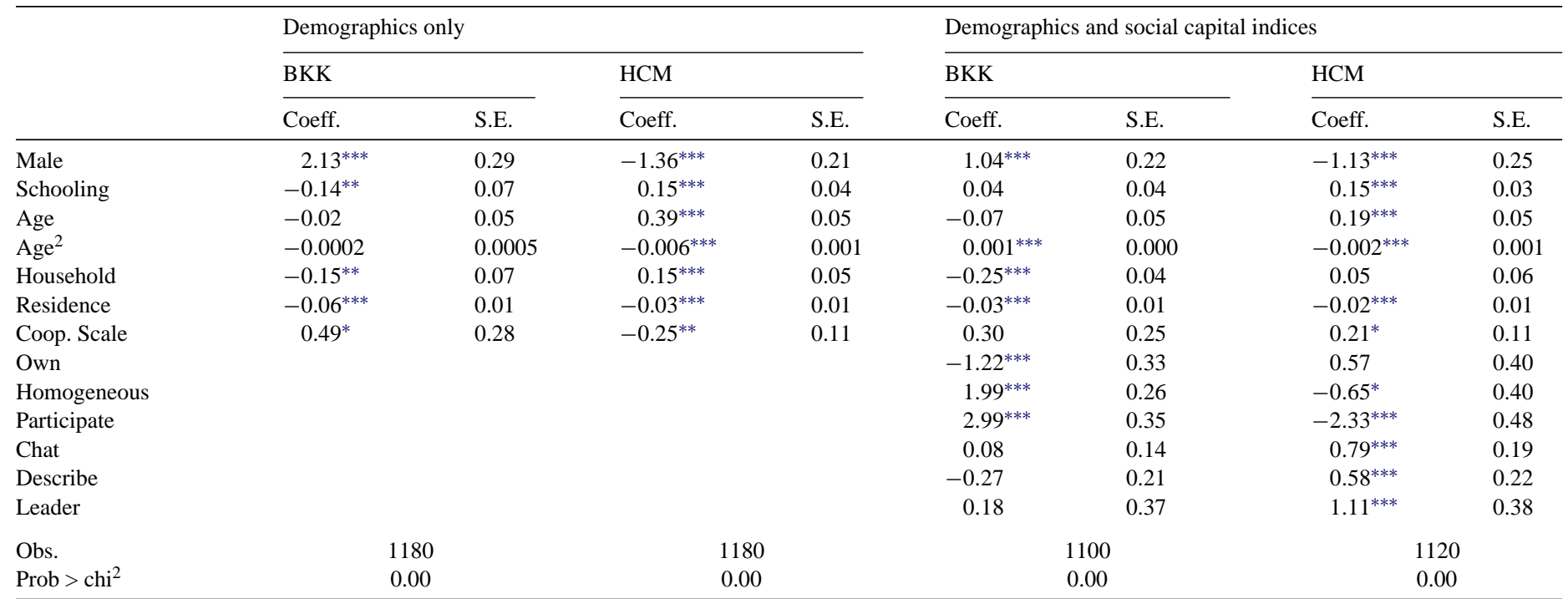

otes: Contributions are bound from below by 0 and from above by 10 .

* Significant at $10 \%$

** Significant at $5 \%$.

*** Significant at $1 \%$ 
contribution by 0.14 coins ( 0.7 Bhat), and there does not appear to be a relationship between age and contributions. However, in an unreported regression that restricts the effect of age to be linear, we find that contributions are significantly decreasing with age (the coefficient is $-0.04, p<0.01$ ). In addition, participants from larger families in Bangkok contribute less, those who have lived in the slums longer contribute less, and those who score higher on the cooperation personality scale contribute significantly more.

Many of the significant effects carry the opposite sign in Ho Chi Minh City. Women contribute more, more schooling leads to higher contributions, and contributions are concave in age with the empirically contribution-maximizing age at 33 years (a result similar to Glaeser et al., 2002). Among the Vietnamese, people from larger households contribute more, but it appears that duration in the slum reduces contributions as in Bangkok.

In the second set of regressions we add the associational variables. The addition of these variables has little effect on many of our demographic results but does add significantly to our estimates. In Bangkok, men remain more cooperative and larger households and long time residents of the slums remain less cooperative, but now the effect of education and the cooperation scale lose significance. Contrary to our priors, home ownership reduces cooperation, but people who perceive their communities to be homogeneous cooperate more (in line with the ingroup hypothesis), and those who claim to have been active in the community also cooperate more. The other coefficients are not significantly different from zero. These results suggest there are some links between network density and behavior in Bangkok.

In Ho Chi Minh City, as in Bangkok, most of the demographic results survive the addition of omitted variables. However, we see that the cooperation personality scale reverses sign, suggesting now that, as we expect, more cooperative types contribute more. Considering the associational variables, we see that, unlike Bangkok, homeownership increases cooperation but not significantly. Interestingly, the Vietnamese react oppositely to homogeneity: people who perceive the slum as more homogeneous contribute less. This result is odd, but might be due to the fact that only $5 \%$ of our players say people mostly come from the same place. It could be that in Vietnam this question is a better measure of feeling like an outsider in a homogeneous community than feeling like an insider. Also, of note is the fact that many of the remaining Vietnamese coefficients are significant while the Thai coefficients were not. People who claim to volunteer for community service contribute less, and those who report chatting with their neighbors more often and describe their neighbors more like family are significantly more cooperative. Lastly, community leaders in Vietnam lead by example: the coefficient is positive and significant.

In terms of the economic significance of these results, compared to the country average contributions, men in Thailand give $15 \%$ more than women, but women in Vietnam give $15 \%$ more than men. A high school education yields no prosocial returns in Bangkok, but in Ho Chi Minh City, this level of educational attainment increases contributions by $24 \%$. At the average duration in the slum, Thais contribute $8 \%$ less and the Vietnamese contribute $6 \%$ less indicating that slum life might attenuate cooperativeness. Homeownership reduces contributions in Bangkok by $18 \%$ while neighborhood homogeneity increases contributions in Bangkok by $30 \%$ and reduces it in Ho Chi Minh City by $9 \%$.

Most of our contribution results are in line with conventional wisdom, but there are two anomalies in the Thai data: homeownership reduces contributions, and men contribute more 
than women. Although the marginal effect is highly significant, we do not put a lot of faith in our home-ownership variable because many of the people who report owning their homes are, in fact, squatting, which means they could never translate their ownership into financial capital and have little incentive to invest in their property. In fact, homeownership, as we understand it in the West, does not really apply to many of the residents in our communities. One could argue that since the implementation of doi moi, however, when the households of Ho Chi Minh City have obtained the power to sell and buy property previously owned by the state, the situation of households in poor neighborhoods has become more like that of the private homeowners of Western Nations (Gainsborough, 2002). On the other hand, the private market has long flourished in Bangkok, leaving many of the poor with little choice but to squat for long periods of time. This also indicates that many of the conventional reasons for ownership to affect social capital (i.e., having a financial stake in the community) apply to few of our respondents.

As it turns out, Thai men have been stereotyped as notorious gamblers. With this information in mind, we thought that one explanation of behavior in Bangkok might be that men were treating our experiment like a slot machine. After a few sessions, we started asking people, at the end of the interview, what the game reminded them of. We expected that if our hypothesis was true that men would disproportionately respond with a comment having to do with gambling. We received six responses from 44 participants: being helpful (14\%), a community project (32\%), nothing (24\%), cooperation (8\%), an investment (18\%), and sports (4\%). Nobody explicitly mentioned gambling, and the distribution of responses by men is actually skewed slightly more towards the cooperative descriptions, although the means (generated by ranking the responses from least to most cooperative) are not significantly different $(t=0.33, p=0.74)$. This suggests that gambling is not the right answer, and men appear to be sincerely more cooperative in Thailand.

In Table 3, we report our social disapproval results. In Vietnam, none of the demographics explain social disapproval; however, one alternative explanation is highly significant. The regressor $\operatorname{Var}($ Cont) is the group-level variance in contributions. We see that the Thais appear immune to the variance in contributions, but the Vietnamese behave as we would expect; when the variance increases (e.g., the group is split between free riders and contributors), the Vietnamese are more likely to show disapproval. Lastly, Contribution is the amount contributed by the individual deciding to signal disapproval or not. In other experiments in which people can punish free riders, we see a strong result between contributing and punishing (e.g., Carpenter, 2002b), but this result does not translate into our social disapproval situation. Cooperators seem just as likely as free riders to disapprove of the group's behavior.

A few additional correlations emerge when we add the associational social capital variables to the Thai regression. Sanctioning remains concave in age, and now duration in the slum predicts disapproval (the longer the duration the more likely participants are to show disapproval), but the effect of household size diminishes. People who perceive the slum to be more homogeneous are less likely to show disapproval, as are those who claim to have participated in a community project. Lastly, the more one chats with her neighbors, the less likely she is to show disapproval. These results suggest that associations dampen one's willingness to speak out against free riding in Thailand. 
Table 3

Dependent variable is one if the individual shows disapproval (results are random effects Logit)

\begin{tabular}{|c|c|c|c|c|c|c|c|c|}
\hline & \multicolumn{4}{|c|}{ Demographics only } & \multicolumn{4}{|c|}{ Demographics and social capital indices } \\
\hline & \multicolumn{2}{|l|}{ BKK } & \multicolumn{2}{|l|}{$\mathrm{HCM}$} & \multicolumn{2}{|l|}{ BKK } & \multicolumn{2}{|l|}{$\mathrm{HCM}$} \\
\hline & M.E. & S.E. & M.E. & S.E. & M.E. & S.E. & M.E. & S.E. \\
\hline Contribution & -0.03 & 0.09 & -0.03 & 0.09 & 0.07 & 0.09 & -0.02 & 0.10 \\
\hline $\operatorname{Var}($ Cont $)$ & 0.01 & 0.05 & $0.14^{* * *}$ & 0.05 & -0.02 & 0.05 & $0.12^{* * *}$ & 0.05 \\
\hline Male & 0.02 & 0.63 & -0.02 & 0.47 & 0.35 & 0.58 & -0.20 & 0.53 \\
\hline Schooling & 0.08 & 0.08 & 0.08 & 0.07 & 0.05 & 0.11 & 0.08 & 0.08 \\
\hline Age & $0.28^{* * *}$ & 0.10 & -0.13 & 0.11 & $0.39^{* * *}$ & 0.15 & -0.09 & 0.12 \\
\hline $\mathrm{Age}^{2}$ & $-0.002^{*}$ & 0.001 & 0.002 & 0.001 & $-0.002^{* *}$ & 0.001 & 0.001 & 0.002 \\
\hline Household & $-0.30^{* * *}$ & 0.11 & 0.02 & 0.09 & -0.19 & 0.12 & 0.07 & 0.11 \\
\hline Residence & 0.03 & 0.02 & 0.02 & 0.02 & $0.06^{* *}$ & 0.02 & 0.02 & 0.02 \\
\hline Coop. Scale & -0.21 & 0.46 & -0.01 & 0.24 & -0.86 & 0.87 & 0.05 & 0.26 \\
\hline Own & & & & & -0.08 & 0.80 & 1.28 & 1.02 \\
\hline Homogeneous & & & & & $-1.36^{* *}$ & 0.54 & 0.13 & 1.27 \\
\hline Participate & & & & & $-3.70^{* *}$ & 1.84 & 0.82 & 1.12 \\
\hline Chat & & & & & $-0.64^{*}$ & 0.37 & $1.28^{*}$ & 0.68 \\
\hline Describe & & & & & 0.82 & 0.54 & 0.003 & 0.51 \\
\hline Leader & & & & & -0.97 & 0.87 & 1.49 & 1.02 \\
\hline Obs. & \multicolumn{2}{|c|}{472} & \multicolumn{2}{|c|}{472} & \multicolumn{2}{|c|}{440} & \multicolumn{2}{|c|}{448} \\
\hline Prob $>\mathrm{chi}^{2}$ & \multicolumn{2}{|c|}{0.00} & \multicolumn{2}{|c|}{0.04} & \multicolumn{2}{|c|}{0.00} & \multicolumn{2}{|c|}{0.16} \\
\hline
\end{tabular}

Notes: Marginal effects are reported.

* Significant at $10 \%$.

** Significant at $5 \%$.

*** Significant at $1 \%$.

The addition of the social capital variables has little effect on the Vietnamese estimate of social disapproval. In fact, the regressors are jointly not different from zero. Again, most of the estimation is driven by the variance in contributions, but there is a marginally significant effect of ties to one's neighbors. The effect is the opposite of what we see in the Thai data. The more contact a Vietnamese player has with her neighbors, the more likely she is to speak out against free riding, indicating that the Vietnamese become more emboldened when they are close to their neighbors.

\section{Comparisons between the Thai and the Vietnamese}

In unreported regressions we also stacked the two data sets from Tables 2 and 3, added Thai dummy variables and generated interactions between the dummies and all our regressors so that we could identify any significant differences between the responses of our Thai participants and their Vietnamese counterparts. In fact, there are a number of interesting results.

In general, chi-squared tests of the joint significance of the demographic differences in both cooperation and social disapproval can not be rejected, nor can similar tests for 
differences in the associational social capital variables. ${ }^{13}$ More specifically, we find that, with respect to our cooperation demographics, Thai men are significantly more cooperative and schooling has a significantly greater effect in Vietnam, as do age and household size. In terms of the effects of associational social capital, Thais are affected significantly differently than Vietnamese when it comes to home ownership, homogeneity, participation in community projects, and their interactions with their neighbors. Thais cooperate more in homogeneous groups while the Vietnamese cooperate less, the Thais cooperate more when they have participated in a community project before, and the Vietnamese cooperate less than the Thais when they seem to have close relationships with their neighbors.

These differences are likely due to cultural and political-economic influences on local cooperation. Homogeneity and communal action among Thai participants reflects the social benefits of strong ties among network members while similar strength of ties among Vietnamese participants may constitute avenues by which monitoring can be effectively maintained. Thus, while homogeneity and neighbor closeness in the Thai context may present opportunities for trust and cooperation, the same may not hold in the more state-controlled Vietnamese communities.

Because the only thing that seems to influence the Vietnamese decision to show disapproval is the variance in group contributions, many of the demographic and social capital estimates are significantly different. Schooling, age, and household size all matter significantly more for the Thais who disapprove more the more schooling they have had, disapprove more and then less as they get older, and disapprove less if they come from larger families.

These cultural differences also exist in the network variables. The Thais are significantly less likely to sanction the group if they claim to own their homes, come from more homogeneous communities, or report having participated in a community project in the last year. However, they are significantly more likely to disapprove if they chat more often with their neighbors. Lastly, as we would expect given the laissez faire nature of Thai culture and the heavy hand of the Vietnamese state, leaders in Vietnam are significantly more likely to signal disapproval.

\section{Discussion}

We contend that the hypothetical nature of many behavioral survey questions used to measure social capital introduces noise and biases that limit the credibility of certain research programs. As a partial solution we conduct experiments in the field with people who live in urban slums where collective action is particularly important to measure behavioral social capital and to see how behavior in the context of monetary incentives varies with demographic factors and associational survey measures of social capital. Overall, we find very high rates of contributions among Thai and Vietnamese participants in a voluntary contribution game and we see that many participants are willing to socially sanction other

\footnotetext{
13 The contribution statistic is $\chi^{2}=202.27$ for the demographic differences and $\chi^{2}=559.26$ for the social capital differences. The disapproval statistics are lower because of the less precise estimates $\left(\chi^{2}=17.37\right.$ for demographics and $\chi^{2}=16.41$ for social capital), but are still significant at the $1 \%$ level.
} 
participants who free ride even though these sanctions are costly to impose and inflict no monetary harm on the targets.

At the individual level, we find that trust and cooperation vary with sex, schooling, age, household size, and years of residence in the slum, as well as placement on a psychological cooperation scale. Additionally, we find that behavior correlates with home ownership, community homogeneity, past participation in community projects, the relationship between players and their neighbors, and community leadership. We also see that a person's willingness to show disapproval of free riding is determined by age, the variance in group level contributions, and a number of our measures of network density. However, many of these correlations differ significantly between our Thai participants and our Vietnamese participants, indicating the critical role of culture and political economic conditions.

Our experiments generate three mysteries: why do the Thai and Vietnamese contribute at high levels, why do participants socially sanction others when this behavior is costly and carries no punch, and why does behavior seem to differ by location? The obvious explanation of the difference in the Thai and Vietnamese contribution rates compared to our Middlebury student controls is that Thai and Vietnamese culture is more cooperative and trusting. In fact there is some broad evidence of East-West differences in cooperation and trust in the experimental literature (Hemesath and Pomponio, 1998; Buchan et al., 2000). One study is particularly relevant: Parks and Vu (1994) compare undergraduates from the United States to recent immigrants from Vietnam in a public goods setting and find, as we do, that the Vietnamese are substantially more cooperative and that the rate of cooperation does not decline with repetition.

The second puzzle concerns why our participants tend to socially sanction the other group members for free riding. We can think of two possible explanations. First, there is considerable work on what has been called expressive voting, which is the idea that people vote to voice their opinions even when they know that the probability that theirs is the determining vote is small (e.g., Brennan and Lomasky, 1993 or Tyran, 2002). It may be the case that our participants understand that there is no cost to the other participants when they express their disapproval, yet they do it anyway because they feel the need to express their moral disapproval.

An alternative, but related explanation, draws on the research described in Carpenter and Matthews (2002) who show that people in a voluntary contribution setting will pay to punish free riders in a completely separate group, even though the punishers can never benefit from higher contributions in the other group. The authors call this behavior social reciprocity and define it as the act of demonstrating one's disapproval, at some personal cost, for the violation of a widely-held norm like not free riding. Our participants' behavior is consistent with social reciprocity because showing disapproval is costly and can not be expected to yield any benefits. Given the prevalence of this behavior in the experimental lab, it is not surprising to also witness it in the field.

Considering our last mystery, can we offer an explanation for the differences we see between our Thai and Vietnamese participants? We think it is plausible to make sense of many of the differences in terms of individualistic versus collectivist cultures defined to include political economy and the insitution of the state (Fukuyama, 1995; Triandis, 1995). Vietnam best resembles a collectivist culture in which many social norms evolve and are supported by punishing deviations from acceptable behavior. At the same time, Thailand is 
usually considered one of the most individualistic cultures in Southeast Asia. Individualistic cultures are characterized as not developing clear norms of behavior and of being more accepting of behavioral differences. These differences are, in fact, reflected in and supported by the governance of both countries (Gainsborough, 2002; Daniere and Takahashi, 2002). Further, for Thais, individualism centers on the individual and household embedded in a hierarchical social system where patrons facilitate access to resources and power for their clients. Consequently, horizontal social relationships (e.g., relations with neighbors) for low-income residents may provide fewer opportunities for social advancement or material improvements than linkages to more powerful patrons (Daniere and Takahashi, 1999).

Overall, our data match this characterization perfectly. Vietnamese players contribute significantly more than Thai players for three reasons. First, the variance of contributions is significantly lower in Vietnam $(p<0.01)$ indicating that players there conform much more than in Thailand. Second, the probability that a Vietnamese player shows disapproval is much higher. We calculated, at the regressor means for each country, the estimated probability that a player who contributes everything when her teammates contribute nothing will signal disapproval. The probability is low in Thailand, 0.08, but it is almost four times higher in Vietnam, 0.29. Third, regressing contributions on the lag of social sanctions shows that disapproval "works" in Vietnam $(\beta=0.79, p<0.01)$ but not in Thailand $(\beta=0.49, p$ $>0.10)$.

Reconsidering section 6 fills in more of the details. For example, consider the fact that schooling makes much more of a difference in Vietnam. A simple explanation of this fact is that culture is partially transmitted through schools (Bowles and Gintis, 1976). In individualistic Thailand, students are not taught specific norms of public cooperation, while in Vietnam, especially considering the role of party ideology, prosocial norms are developed and strongly reinforced in school (Daniere and Takahashi, 1999). Similarly, it is interesting that leaders only make a difference among the Vietnamese. Thai leaders follow disparate norms, but Vietnamese leaders contribute to the good of the group. Notice that in Thailand the likelihood of disapproving increases in age for our sample, which we think is consistent with the fact that in many cultures, norms are enforced by the older members of communities. Recall, however, that age does not matter in Ho Chi Minh City because here the party and stricter government control may substitute for elders in the enforcement of prosocial behavior. Moreover, we see that the only thing that matters to the Vietnamese when it comes to disapproval is the variance in contributions, which is consistent with collectivist culture and the notion of social reciprocity; if there are too many free riders breaking the norm, they are sanctioned, regardless of the specifics of the situation. The significance of many more of the regressors in Thailand indicates that there are many more "types" of people who show disapproval for different reasons.

\section{Acknowledgment}

We thank Anchana NaRanong (NIDA, Bangkok), Nguyen Quang Vinh, and Van Thi Ngoc Lan (ISSHO, HCM City) for their help in conducting this research. We also thank Iris Bohnet, Rachel Croson, Jon Isham, Peter Matthews, Bob Slonim, Jean-Robert Tyran, Chris Winship, two anonymous referees, and the participants in the 2003 Trust and Institutions 
seminar at the Kennedy School of Government for comments. Support for this project came from the UCal. Pacific Rim Research Program, the Social Science and Humanities Research Council of Canada, and the NSF (SES-CAREER 0092953).

\section{References}

Ahn, T.K., Ostrom, E., Schmidt, D., Walker, J., 2003. Trust in two-person games: games structures and linkages. In: Ostrom, E., Walker, J. (Eds.), Trust and Reciprocity: Interdisciplinary Lessons from Experimental Research. Russell Sage Foundation, New York, pp. 323-351.

Ashraf, N., Bohnet, I., Piankov, N., 2003. Decomposing trust. JFK School Working paper.

Berg, J., Dickaut, J., McCabe, K., 1995. Trust, reciprocity and social history. Games and Economic Behavior 10, $122-142$.

Bertrand, M., Mullainathan, S., 2001. Do people mean what they say? Implications for subjective survey data. American Economic Review 91 (2), 67-72.

Bowles, S., Gintis, H., 1976. Schooling in Capitalist America: Educational Reforms and the Contradictions of Economic Life. Basic Books, New York.

Bowles, S., Gintis, H., 2002. Social capital and community governance. The Economic Journal 112 (483), 419-436.

Brennan, G., Lomasky, L., 1993. Democracy and Decision: The Pure Theory of Electoral Preference. Cambridge University Press, Cambridge.

Buchan, N., Croson, R., Johnson, E., 2000. Trust and reciprocity: an international experiment. School of Business Working paper, University of Wisconsin, Madison.

Cardenas, J.C., Carpenter, J., 2002. Using cross-cultural experiments to understand the dynamics of a global commons. Middlebury College Center for International Affairs Working paper 2.

Carpenter, J., 2002a. Measuring social capital: adding field experimental methods to the analytical toolbox. In: Isham, J., Kelly, T., Ramaswamy, S. (Eds.), Social Capital and Economic Development: Well-Being in Developing Countries. Edward Elgar, Northampton, pp. 119-137.

Carpenter, J., 2002. Punishing free-riders: how group size affects mutual monitoring and collective action. Middlebury College Department of Economics Working paper 2002-06.

Carpenter, J., Matthews, P., 2002. Social reciprocity. Middlebury College Department of Economics Working paper 2002-29.

Carter, M., Castillo, M., 2002. The economic impacts of altruism, trust and reciprocity: an experimental approach to social capital. AAE Staff papers, University of Wisconsin, Madison.

Croson, R., Buchan, N., 1999. Gender and culture: international experimental evidence from trust games. American Economic Review (papers and proceedings) 89 (2), 386-391.

Daniere, A., Takahashi, L., 1999. Environmental behavior in Bangkok, Thailand: a portrait of attitudes, values, and behavior. Economic Development and Cultural Change 47 (3), 525-557.

Daniere, A., Takahashi, L., 2002. Rethinking Environmental Management in the Pacific Rim: Exploring Local Participation in Bangkok, Thailand. Ashgate Publishing Ltd., Hampshire, United Kingdom.

Daniere, A., Takahashi, L., NaRanong, A., 2002. Social capital, networks and community environments in Bangkok, Thailand. Growth and Change 33 (4), 453-484.

Desdoigts, A., 1999. Patterns of economic development and the formation of clubs. Journal of Economic Growth 4 (3), 305-330.

Deutsch, M., 1973. The Resolution of Conflict: Constructive and Destructive Processes. Yale University Press, New Haven.

Drakakis-Smith, D., Dixon, C., 1997. Sustainable urbanization in Vietnam. Geoforum 28 (1), 21-38.

Durlauf, S.N., 2002. On the empirics of social capital. The Economic Journal 112 (483), 459-479.

Fritzen, S., 2002. Growth, inequality and the future of poverty reduction in Vietnam. Journal of Asian Economies $13(5), 635-657$.

Fukuyama, F., 1995. Trust: The Social Virtues and the Creation of Prosperity. The Free Press, New York.

Gächter, S., Herrmann, B., Thoeni, C, 2003. Trust, voluntary cooperation, and socio-economic background: survey and experimental evidence. Journal of Economic Behavior \& Organization 55 (4), 505-531. 
Gainsborough, M., 2002. Understanding communist transition in Ho Chi Minh City in the late 1990s. PostCommunist Economics 14, 227-243.

Glaeser, E., Laibson, D., Sacerdote, B., 2002. The economic approach to social capital. The Economic Journal 112 (Nov.), 437-458.

Glaeser, E., Sacerdote, B., 1999. The social consequences of housing. Journal of Housing Economics 9 (1-2), 1-23.

Güth, W., Ockenfels, P., Wendel, M., 1997. Cooperation based on trust: an experimental investigation. Journal of Economic Psychology 18, 15-43.

Hemesath, M., Pomponio, X., 1998. Cooperation and culture: students from China and the United States in a prisoner's dilemma. Cross-Cultural Research: The Journal of Comparative Social Science 32 (2), 171-184.

Henrich, J., Boyd, R., Bowles, S., Camerer, C., Fehr, E., et al., 2001. In search of homo economics: behavioral experiments in 15 small-scale societies. American Economic Review 91 (2), 73-78.

Isham, J., Kelly, T., Ramaswamy, S., 2002. Social capital and well-being in developing countries: an introduction. In: Isham, J., Kelly, T., Ramaswamy, S. (Eds.), Social Capital and Economic Development: Well-Being in Developing Countries. Edward Elgar, Northampton, pp. 3-17.

Kakwani, N., Krongkaew, M., 2000. Analyzing poverty in Thailand. Journal of the Asian Pacific Economy 5 (1/2), $141-160$.

Kim, W.B., Douglass, M., Ho, K.C., 1997. Culture and the City in East Asia. Oxford University Press, Oxford.

Knack, S., Keefer, P., 1997. Does social capital have an economic payoff? A cross-country investigation. Quarterly Journal of Economics 112, 1251-1288.

Luong, H.V., 2003. Economic growth and governances transformation in Vietnam. Department of Geography and Planning Working paper, University of Toronto.

McCabe, K., Rassenti, S., Smith, V., 1996. Game theory and reciprocity in some extensive form experimental games. Proceedings of the National Academy of Science USA 93 (Nov.), 13421-13428.

Messick, D., Mackie, D., 1989. Intergroup relations. In: Rosenzweig, M., Porter, L. (Eds.), Annual Review of Psychology. Annual Reviews Inc, Palo Alto, CA, pp. 45-81.

Messick, D.M., Wilke, H., Brewer, M., Kramer, R., Zemke, P., et al., 1983. Individual adaptations and structural change as solutions to social dilemmas. Journal of Personality and Social Psychology 44, 294-309.

Parks, C., Hulbert, L., 1995. High- and low-trusters' responses to fear in a payoff matrix. Journal of Conflict Resolution 39, 718-730.

Parks, C., Vu, A., 1994. Social dilemma behavior of individuals from highly individualist and collectivist cultures. Journal of Conflict Resolution 38 (4), 708-718.

Putnam, R.D., 2000. Bowling Alone: The Collapse and Revival of American Community. Simon and Schuster, New York.

Sampson, R., Raudenbush, S., Earls, F., 1997. Neighborhoods and violent crime: a multilevel study of collective efficacy. Science 277 (Aug. 15), 918-924.

Setchell, C, 1992. Final Report of the Greater Bangkok Slum Housing Market Study. Regional Housing and Urban Development Office, USAID, Bangkok.

Singh, D., Freeman, N., 2001. Regional outlook: Southeast Asia 2001-2002. Institute of Southeast Asian Studies, Singapore.

Triandis, H., 1995. Individualism and Collectivism. Westview Press, Boulder.

Tyran, J.-R., 2002. Voting when money and morals conflict: an experimental test of expressive voting. Department of Economics Working paper 2002-07, University of St. Gallen.

Woolcock, M., 1995. Social capital and economic development: towards a theoretical synthesis and policy framework. Theory and Society 27, 151-208. 